\section{ORIGINAL RESEARCH}

\author{
P. Niggemann \\ M. Seifert \\ A. Förg \\ H.H. Schild \\ H. Urbach \\ T. Krings
}

\section{Positional Venous MR Angiography: An Operator- Independent Tool to Evaluate Cerebral Venous Outflow Hemodynamics}

BACKGROUND AND PURPOSE: CCSVI has been proposed as a cause for MS. According to this theory, strictures of the IJV are among the described causes for CCSVI. Little is known about their influence on the hemodynamics of the CVBO. We used positional MR imaging to describe the influence of positional changes on the CVBO.

MATERIALS AND METHODS: Using the Fonar Upright MR imaging system, we performed venous time-of-flight angiography of the cervical region in the supine and sitting positions in 15 healthy volunteers. The image quality was rated; the positional findings and interindividual variances in the CVBO were analyzed.

RESULTS: A venous time-of-flight angiography of the cervical spine was feasible with good image quality. Strictures of 1 or both IJVs were found in 8 of 15 healthy volunteers in the supine position; however, none were visible in upright position. The IJV was not the main outflow route in the erect position. No relevant venous reflux was observed.

CONCLUSIONS: IJV strictures are a common finding in healthy volunteers in the supine position. They seem to be of no relevance in the erect position. This finding questions the validity of this criterion for the diagnosis of CCSVI. Reflux into the venous system was not visualized, and it remains to be seen whether it can be identified in patients with MS. Positional MR imaging enables operator-independent evaluation of the CVBO and may help to clarify the validity of the criteria for CCSVI.

ABBREVIATIONS: CCSVI = chronic cerebrospinal venous insufficiency; $\mathrm{CVBO}=$ cerebral venous blood outflow; EJV = external jugular vein; IJV = internal jugular vein; MIP = maximum intensity projection; NV = nuchal veins; $V P=$ veins of the vertebral plexus
$\mathbf{T}$ he theory that MS is an inflammatory demyelinating disorder of the central nervous system most likely related to an autoimmune disorder has been questioned recently by Zamboni et al. ${ }^{1}$ These authors suggested that an impairment of the CVBO, such as strictures of the IJV or position-dependent venous reflux, led to CCSVI. CCSVI may lead to iron deposition along the cerebral veins, activation of the complement system, and macrophage-activated demyelination, leading to MS. ${ }^{1}$

Some groups confirmed certain findings of Zamboni et al, ${ }^{1}$ but the implications of venous strictures still remain unclear. $^{2-5}$

Different body postures have different patterns of cerebral venous outflow, and a considerable variation of the cerebral venous outflow has been reported. ${ }^{6-8}$ Methods, like duplex sonography or conventional MR venography, which are routinely used in the clinical setting to evaluate the cerebral venous outflow, have significant drawbacks when trying to evaluate position-dependent changes.

Positional MR imaging was recently introduced as a new possibility to image the CVBO in different postures. ${ }^{9,10}$ The presented methods, however, do not allow investigating stric-

Received March 22, 2011; accepted after revision June 8

From the Department of Radiology (P.N., M.S., H.H.S., H.U.), University of Bonn, Bonn, Germany; Privatpraxis für Upright MRT (A.F.), Aschheim, Germany; and Department of Neuroradiology (T.K.),Toronto Western Hospital, Toronto, Ontario, Canada.

Please address correspondence to Pascal Niggemann, MD, Department of Radiology, University of Bonn, Sigmund-Freud Str 25, 53127 Bonn, Germany; e-mail: p.niggemann@mrt-koeln.de

http://dx.doi.org/10.3174/ajnr.A2774 tures, since they either lack the possibility to acquire 3D datasets or do not cover the entire cervical region.

By using positional MR imaging, we report a venous timeof-flight sequence allowing the acquisition of $3 \mathrm{D}$ datasets covering the entire cervical region. The usefulness of the sequence and the variability of the major cerebral venous outflow system in different body positions are examined, and findings are discussed in regard to the proposed criteria for CCSVI.

\section{Materials and Methods}

Fifteen consecutive healthy volunteers (9 women, 6 men; mean age, 34 years of age) without any history of neurologic disorders were prospectively recruited for this study. After obtaining written consent, we imaged the CVBO in the supine and sitting positions by using the Fonar Upright MR imaging system (Fonar, Melville, New York), a water-cooled resistive $0.6 \mathrm{~T}$ open magnet using a single-channel cervical ring coil.

A scout image was obtained to identify the segments of the cervical spine. We acquired a 2D time-of-flight sequence, allowing the acquisition of 3D datasets covering the craniocervical junction, the cervical spine, and the upper thoracic spine (to T2) (TE, $12 \mathrm{~ms}$; TR, $45 \mathrm{~ms}$; flip angle, $30^{\circ}$; matrix, $200 \times 200$; FOV, $22 \times 22 \mathrm{~cm}$; section thickness, 3.5 $\mathrm{mm}$; section spacing, $1.1 \mathrm{~mm}$; total scan coverage, $176 \mathrm{~mm}$; scan direction, cranial to caudal; time of acquisition, 21 minutes, $45 \mathrm{sec}-$ onds). An arterial saturation pulse was applied. Section angulations of $90^{\circ}$ to the midcervical spine were chosen, and the lowest part of the image stack was placed in the upper third of the T2 vertebral body. The sequence was always planned in the same fashion and obtained in such a manner that only blood flowing from the head to the heart 


\begin{tabular}{|c|c|c|c|c|c|c|c|c|}
\hline \multirow[b]{2}{*}{ Volunteer } & \multicolumn{4}{|c|}{ Supine } & \multicolumn{4}{|c|}{ Sitting } \\
\hline & IJV & EJV & VP & NV & IJV & EJV & VP & NV \\
\hline 1 & + & + & - & + & - & + & + & + \\
\hline 2 & + & + & - & - & - & + & + & + \\
\hline 3 & + & + & + & + & - & - & + & + \\
\hline 4 & + & - & + & + & + & + & + & - \\
\hline 5 & + & + & + & - & + & - & + & + \\
\hline 6 & + & + & - & + & $(+)$ & - & + & + \\
\hline 7 & + & - & + & + & + & - & + & + \\
\hline 8 & + & + & - & - & + & - & - & + \\
\hline 9 & + & + & - & - & $(+)$ & - & + & - \\
\hline 10 & + & + & - & + & + & + & + & + \\
\hline 11 & + & + & - & + & + & - & - & + \\
\hline 12 & + & + & - & - & $(+)$ & - & + & - \\
\hline 13 & + & + & + & - & $(+)$ & - & + & + \\
\hline 14 & + & + & - & - & $(+)$ & - & + & + \\
\hline 15 & + & + & - & - & $(+)$ & - & + & + \\
\hline
\end{tabular}

Note: + indicates vessel delineated; -, vessel not delineated; in parentheses, delineation of the IJV only on 1 side.

yielded signal intensity. The dataset was reformatted by the scanner software, and 3D MIP images were obtained.

The images were analyzed digitally by using PACS software (JiveX, Visus, Bochum, Germany).

We recorded the following points: The covered scan field was divided into 3 parts: the craniocervical junction (from the brain to $\mathrm{C} 1)$, the cervical spine ( $\mathrm{C} 1 / 2$ to $\mathrm{C} / \mathrm{T} 1)$, and the upper thoracic spine (T1 and T2). The division into these 3 parts was chosen because of the expected differences in image quality. The image quality for each segment was assessed by the 2 senior neuroradiologists and rated according to the following scale: good, acceptable, poor, and nondiagnostic.

The main venous outflow route for the supine and sitting positions was determined in consensus. Veins contributing to the CVBO, postural variations, and the presence or absence of high-grade venous strictures of the IJV were recorded. Strictures were divided into not significant $(<90 \%$ reduction of the diameter in regard to the poststenotic part of the vein) and high-grade $(>90 \%)$. The location of the stricture was noted. A $\kappa$ test was performed to assess the interobserver variability of the image quality (Analyze-it; Analyze-it Software, Leeds, UK).

\section{Results}

The depiction of the venous system was possible in all volunteers by using venous $2 \mathrm{D}$ time-of-flight angiography both in the supine and erect positions. Only venous flow signal intensity was detected, and in neither position was an arterial flow signal intensity found.

The image quality varied in the 3 defined segments as follows: At the craniocervical junction, the image quality was acceptable in 12 volunteers and graded as nondiagnostic, poor, and good in 1 patient each. At the level of the cervical spine, the image quality varied between good (12 volunteers) and acceptable (3 volunteers). At the level of the upper thoracic spine, the image quality was rated as nondiagnostic for all volunteers by both readers. Both readers assigned the same image-quality score for all volunteers and all segments, leading to a $\kappa$ value of 1 .

Table 1 shows the delineation of the IJV and collateral vessels in both positions. A great variability of the cerebral blood outflow tract was noted (Figs 1-4). In the supine position, additional vessels besides the IJV contributing to the cerebral venous outflow could be identified in all volunteers (Figs 1-4). The EJV was visible in 13 volunteers; the VP, in 5 volunteers; and the NV, in 7 volunteers.

In the sitting position, a marked change of the delineated venous outflow was noted. The IJV was only seen in 6 volunteers on both sides and in another 6 volunteers on 1 side. In 4 volunteers the EJVs, in 13 volunteers the VP, and in 12 volunteers the NV were identified contributing to the CVBO.

Table 2 shows the delineation of the IJV and the presence or absence of strictures. A so-called "normal" CVBO pattern without any significant narrowing of 1 or 2 of the IJVs in the supine position was only seen in 7 volunteers (Figs 1 and 2). The other 8 volunteers had significant strictures of at least 1 IJV in the superior portion (Figs 3 and 4). In 3 of these 8 volunteers with a significant stenosis of the IJV in the supine position, the IJV was only delineated in the sitting position on both sides and in 2 volunteers on 1 side. It was, however narrowed compared with the findings in the supine position. The narrowing was homogeneous, and no stricture in the course of the IJV was observed. In the remaining 3 volunteers with a significant stenosis of the IJV in the supine position, the IJV was not delineated in the sitting position.

\section{Discussion}

\section{Technical Features of Positional MR Venography}

Reports on positional venous MR angiography are scarce,, 10 because only a few dedicated MR imaging scanners are available. The magnetic field of positional MR imaging scanners is typically lower compared with conventional MR imaging scanners; hence the possibility of performing MR angiography is reduced. However, even in such low-magnetic-field scanners, a 2D time-of-flight angiography can be acquired. The use of 2D time-of-flight angiography enables depiction of slowflowing blood, which is required for an optimal visualization of the venous system. The presented time-of-flight angiography yields good-quality images, and the entire cerebral venous outflow system can be depicted.

A limitation of the technique, however, is the prolonged scanning time, which is also problematic in terms of sensitivity to patient movements. With 21 minutes, 45 seconds for 1 sequence, the total scanning time is long, and patients or volunteers tend to move, especially in the sitting position. In our study, slight motion artifacts of the volunteers did not influence the image quality greatly; however, whether images of equal quality can be acquired in patients with MS is still unknown. The distance covered by the sequence is limited to 176 $\mathrm{mm}$, which allowed visualizing the entire cervical region in our volunteers. However, this may not be sufficient in tall patients for depicting the entire cerebral venous outflow system.

The image quality of our venous time-of-flight angiography was satisfactory at the level of the craniocervical junction and the cervical spine. The reduction of signal-intensity quality at the cervicothoracic junction and the thoracic part of the jugular and vertebral veins can be explained by coil performance. The ring coil lies loose around the neck; hence, the area of maximal signal intensity is the cervical region. The signal 


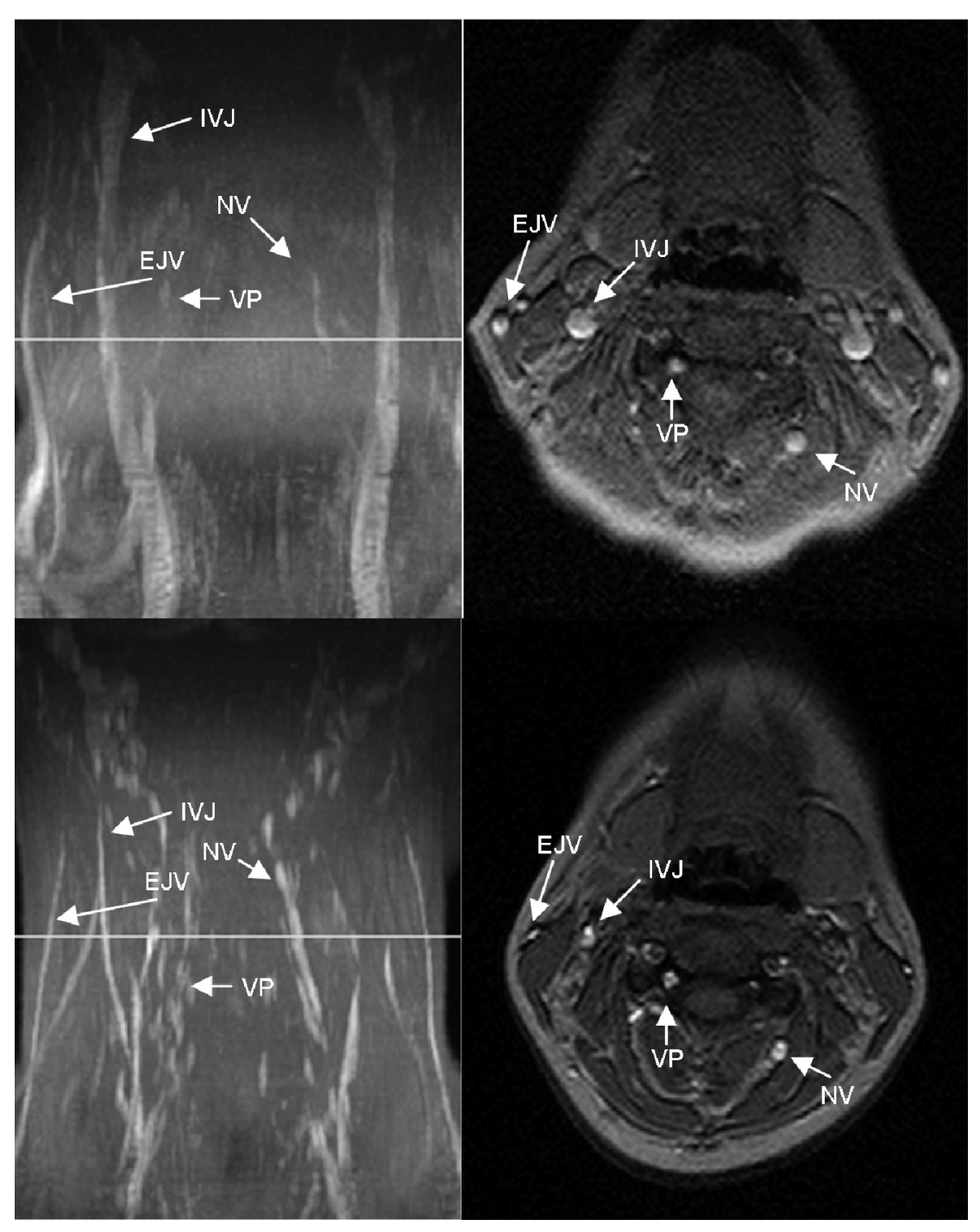

Fig 1. MIP (left column) and axial source images at the level of $\mathrm{C} 4$ in the supine (top row) and sitting positions (bottom row) in a healthy volunteer. In the supine position, there is no significant narrowing of the IJV. The EJV, NV, and the VP can be identified in the supine position. In the sitting position, the IJV, EJV, and the NV are much less prominent, whereas the $V P$ is slightly more prominent.

intensity is, therefore, reduced at the craniocervical and the upper thoracic spine. The more turbulent blood flow at the craniocervical junction does not seem to greatly influence the image quality, and the signal intensity is still adequate. At the level of the thoracic spine, the image quality is severely reduced due to respiratory movement artifacts, and no adequate images could be acquired by using the presently available technology.

Even with these limitations, the presented 2D time-offlight angiography seems useful to investigate the CVBO, especially in regard to the cause of CCSVI.

\section{Usefulness of Positional MR Venography in Regard to CCSVI Criteria}

Zamboni et al ${ }^{1}$ described 5 duplex sonography criteria that are considered highly suspicious for CCSVI. All patients with MS in his study met at least 1 or 2 criteria, whereas these findings were never encountered in his control population. ${ }^{11}$ These findings have already been questioned by other groups and were not always reproducible. ${ }^{2,4}$ In detail, the criteria presented by Zamboni et $\mathrm{al}^{1}$ were the following: 1) reflux in the IJV or vertebral veins in the sitting or supine position, 2) reflux in the deep cerebral veins, 3) proximal (superior) stricture of the IJV, 4) lack of Doppler-detectable flow in the IJV or verte- bral veins, and 5) reverted postural control of the main cerebral venous outflow pathway.

Because these criteria were defined for duplex sonography, whether they can be investigated by MR imaging remains to be seen. MR imaging and duplex sonography use different physical properties to image the human body and physiologic processes. Although duplex sonography yields high resolution in time and space, it is operator-dependent and limited by bone structures and its 3D abilities are not optimal. Pressure applied to compressible veins may also influence findings.

MR imaging, on the other hand, allows reproducible $2 \mathrm{D}$ or $3 \mathrm{D}$ images with very high tissue contrast. It lacks, however, the temporal resolution of sonography. Also, there is no pressure applied to the veins during the examination.

A stricture of the jugular veins can be observed easily by using positional MR imaging, and we could find a high number of unilateral and bilateral strictures in volunteers. The proposed criterion 3 can hence be imaged by positional MR imaging without any restrictions.

Unlike contrast-enhanced or phase conventional angiography, time-of-flight angiography is only sensitive to unidirectional blood flow. Because no arterial flow signal intensity is visible, the presented venous time-of-flight angiography seems to be sensitive only for blood flowing in the craniocau- 
Fig 2. MIP (left column) and axial source images at the level of $\mathrm{C} 2$ in supine (top row) and sitting positions (bottom row) in a healthy volunteer. In the supine position, there is a slight narrowing of the IJVs. The EJVs, the NV, and VP can be identified in the supine position. In the sitting position, IJVs and EJVs are no longer visible, whereas the NV are much more prominent. The VP can be identified.

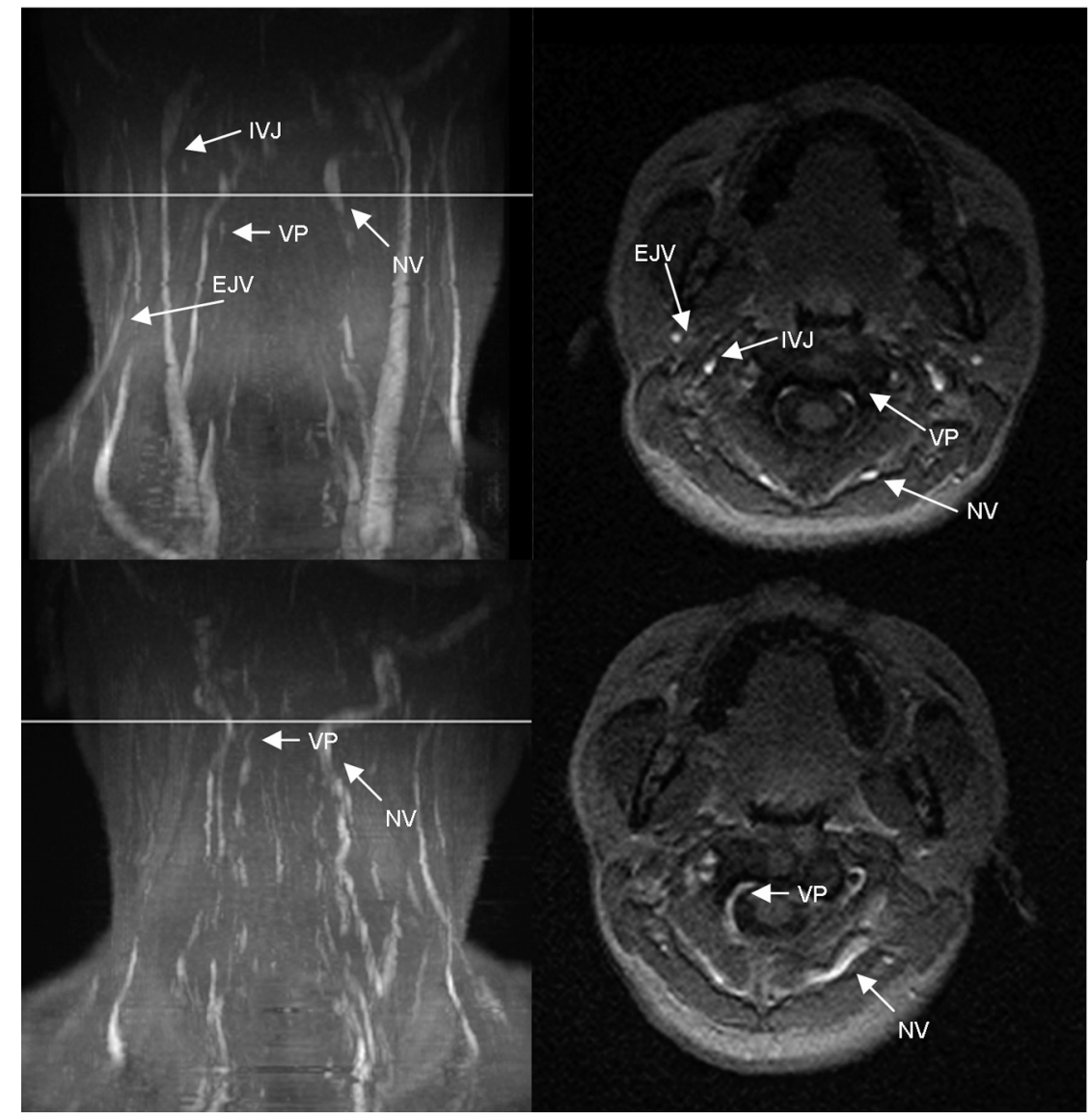

Fig 3. MIP (left column) and axial source images at the level of C2 in supine (top row) and sitting positions (bottom row) in a healthy volunteer. In the supine position, there is a high-grade stricture of the left jugular vein (arrow). The EJV and VP are visible. In the sitting position, only the right IJV is visible (arrowhead). The NV are prominent, and the VP can be identified.
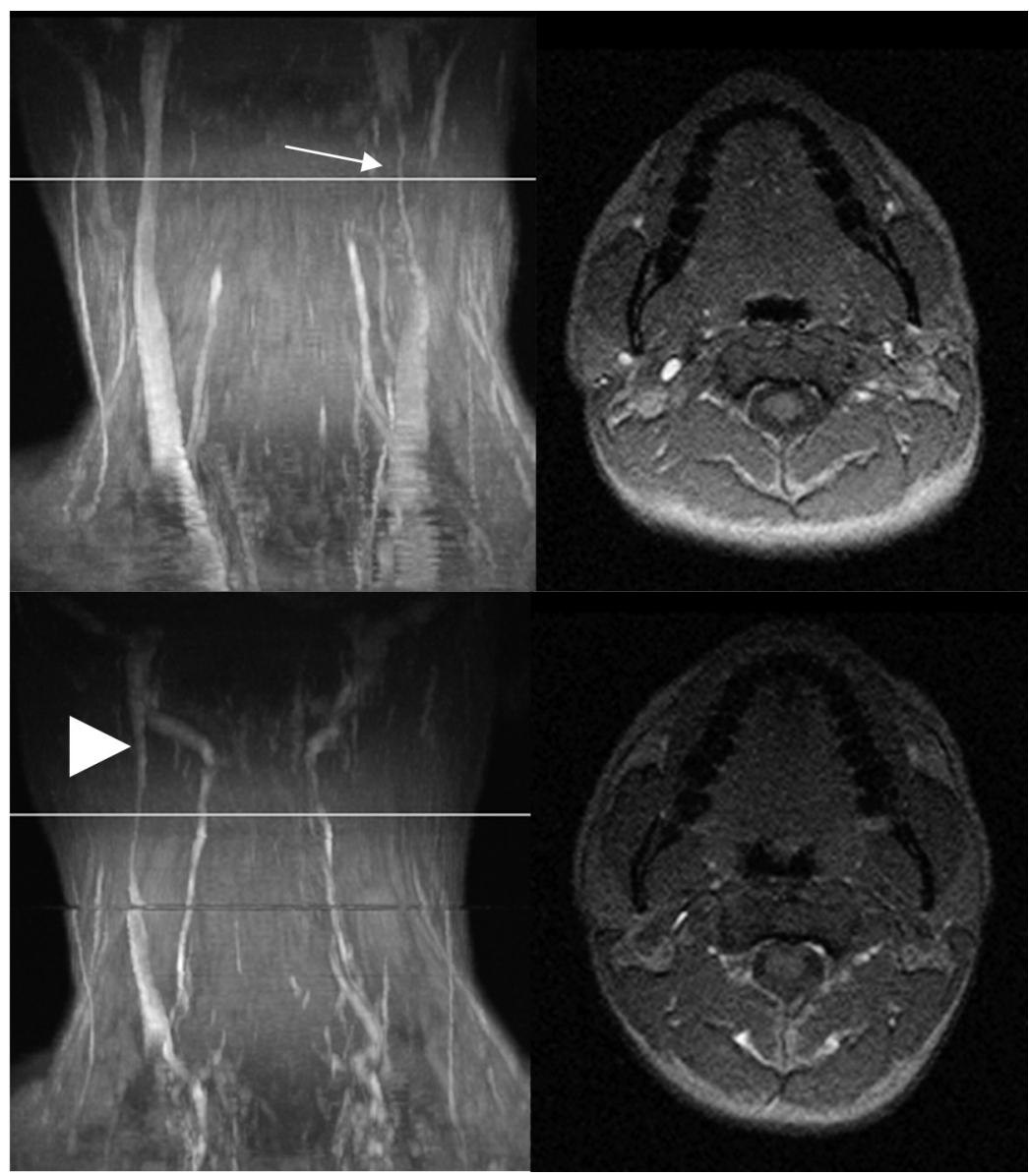


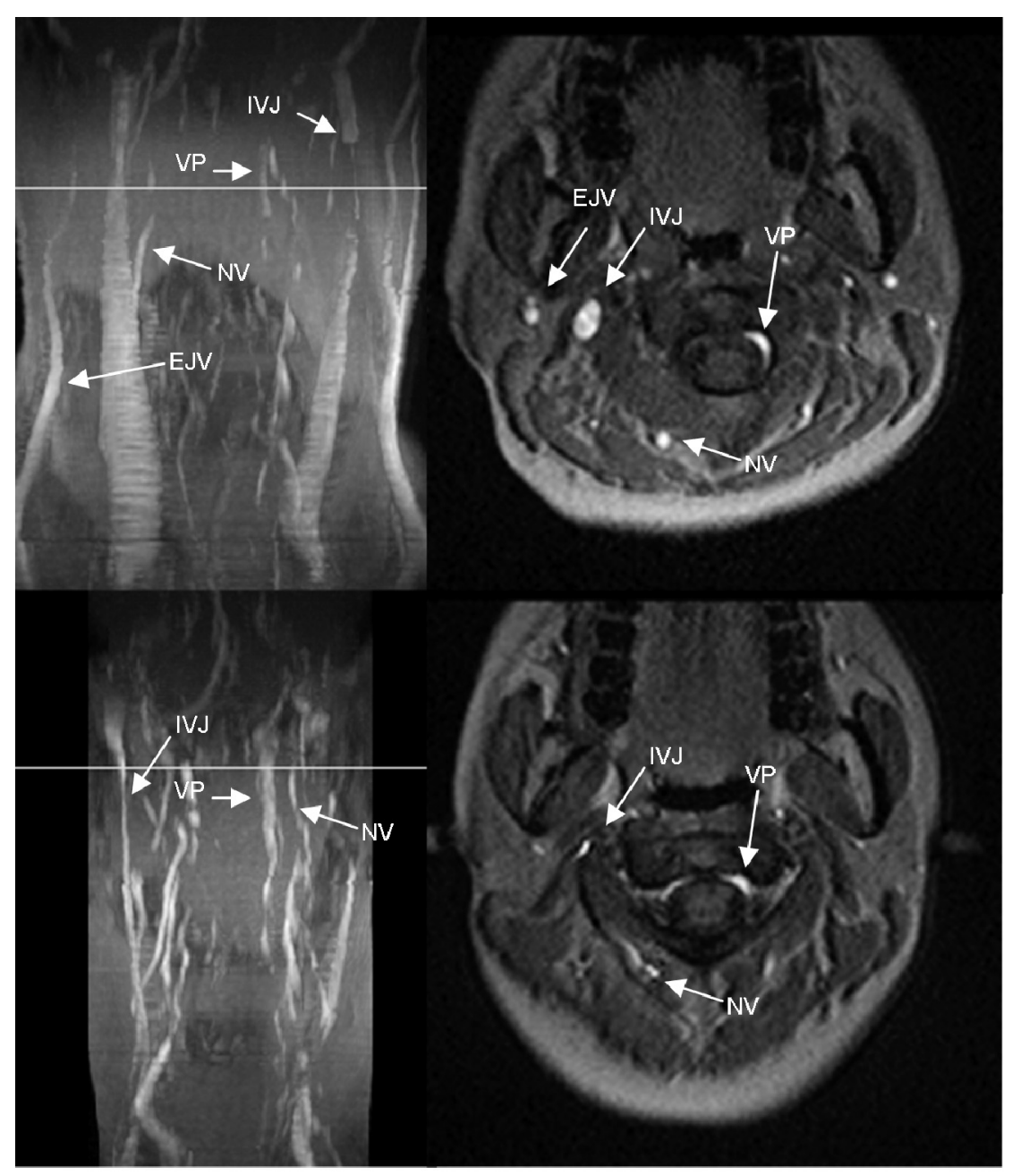

Fig 4. MIP (left column) and axial source images at the level of $\mathrm{C} 2$ in supine (top row) and sitting positions (bottom row) in a healthy volunteer. In the supine position, there is a high-grade stricture of the left IJV. The EJV, NV, and VP are visible. In the sitting position, only the right IJV is visible, but narrowed. The VP are prominent, and the NV can be identified.

Table 2: The delineation of venous strictures in the IJV and the localization of the strictures for all volunteers

\begin{tabular}{llllc}
\hline & \multicolumn{2}{c}{ Supine } & & \multicolumn{2}{c}{ Sitting } \\
\cline { 2 - 4 } Volunteer & IJV Delineation & Occurrence of Stricture & & Occurrence of Stricture \\
\hline 1 & Bilateral & Both sides superior & Not depicted & NA \\
2 & Bilateral & Both sides superior & Not depicted & NA \\
3 & Bilateral & Left side superior & Not depicted & NA \\
4 & Bilateral & No stricture & Bilateral & Narrowed, no stricture \\
5 & Bilateral & No stricture & Bilateral & Narrowed, no stricture \\
6 & Bilateral & No stricture & Right side only & Narrowed, no stricture \\
7 & Bilateral & Left side superior & Bilateral & Narrowed, no stricture \\
8 & Bilateral & No stricture & Bilateral & Narrowed, no stricture \\
9 & Bilateral & No stricture & Left side only & Narrowed, no stricture \\
10 & Bilateral & Right side superior & Bilateral & Narrowed, no stricture \\
11 & Bilateral & Both sides superior & Bilateral & Narrowed, no stricture \\
12 & Bilateral & No stricture & Right side only & Narrowed, no stricture \\
13 & Bilateral & Both sides superior & Right side only & Narrowed, no stricture \\
14 & Bilateral & No stricture & Right side only & Narrowed, no stricture \\
15 & Bilateral & Right side superior & Right side only & Narrowed, no stricture \\
\hline
\end{tabular}

Note:-NA indicates not applicable.

dal direction. Therefore, a reflux in the IJV or cerebral veins should have been detectable as a lack of signal intensity in the presence of a stricture; however, no such lack of signal intensity was observed. Technically, criteria 1 and 2 can hence be evaluated by positional MR imaging. This, however, has to be confirmed in patients with MS with refluxes proved by duplex sonography.
Our findings confirm that the cerebral venous blood flow varies between the supine and erect position and that to evaluate the CVBO, flow in all vessels contributing to the cerebral venous outflow must be considered. The lack of flow in the cervical veins, criterion 4 , can be investigated by using positional MR imaging.

Reverted postural control of the main cerebral venous out- 
flow pathway, criterion 5, is defined as an increase in diameter of the IJV in the sitting position compared with the supine position. Our findings show that this can be demonstrated by positional MR imaging. As opposed to duplex sonography, positional MR imaging can show the whole venous vasculature in a reproducible way and, therefore, seems adequate to show postural changes without the limitations of duplex sonography.

\section{Positional MR Imaging Findings in Healthy Volunteers}

As reported previously, the cerebral venous outflow is dependent on the position of the subject. In the supine position, the IJVs are the main outflow route for the cerebral blood. In the erect position, the IJVs collapse because of their position above the heart, leading to negative pressure in the IJV. ${ }^{6}$ The main cerebral blood outflow routes in this position are the VP and the NV. ${ }^{6,7,12}$

Significant interindividual variances in the vessel size and vessel recruitment for the cerebral blood outflow were found. The variability is not surprising because cervical veins show multiple interconnections. ${ }^{8,13}$ Unlike head and neck arteries, which are anatomically well-defined vessels, the veins of the vertebral venous plexus contributing to the cerebral blood outflow are less defined vessels and show multiple interconnections. ${ }^{14} \mathrm{~A}$ defined vertebral vein is not present at the level of the upper and middle cervical spine and is only visible at the cervicothoracic junction. ${ }^{9}$

More than half of the volunteers in this study had strictures of the superior jugular vein; hence, it seems to be an anatomic variation rather than a truly relevant abnormal finding in the general population. In the erect position, none of the visible jugular veins showed any strictures. Therefore, it seems unlikely that strictures of the IJV found in the supine position have any hemodynamic influence. Reports on patients with bilateral thrombosis of the jugular veins showed that vertebral venous plexuses are elastic vessels, which might dilate in case of an outflow obstacle in other parts of the cerebral venous drainage system. ${ }^{15}$ We did not observe more prominent or more frequent VP in volunteers with strictures compared with volunteers without strictures.

Our findings suggest that because of the variability of the $\mathrm{CVBO}$, the findings in the erect position cannot be predicted from the findings in the supine position.

\section{Clinical Implications in Regard to CCSVI}

Our findings in the healthy population suggest that because of the variability of the cerebral venous outflow system, it is unlikely that a stricture of 1 or both jugular veins can lead to venous congestion of the brain.

The CVBO shows high interindividual variability and abundant collaterals at the level of the cervical spine. Strictures of 1 or both jugular veins are common in the healthy population and are unlikely to be of any clinical significance, given the above-mentioned abundant collateral network. One may, therefore, presume that for intracranial venous congestion to arise, additional outflow obstructions of the head and neck venous system are necessary.

The findings of Zamboni et $\mathrm{al}^{16}$ led them and others to perform angioplasty of the strictures of the IJV. Our findings suggest that before dilating strictures of the IJV, the entire venous system has to be evaluated and the hemodynamic relevance of the strictures on CVBO has to be confirmed.

To clarify the role of the cervical venous system in the development or aggravation of multiple sclerosis, more research is necessary.

The model presented by Zamboni et $\mathrm{al}^{1,11}$ seems to oversimplify the rather complex venous drainage of the brain and its dependence on positioning. An operator-independent method such as the one proposed in this study may help clarify this controversial topic. Hence, a study on the correlation of positional MR findings with duplex sonography findings in patients with MS would be useful.

Strictures of the jugular veins are common in healthy subjects. Our findings suggest that uni- or bilateral strictures do not impair the cerebral blood outflow. Positional MR imaging is a promising tool to show the cerebral blood outflow and is suitable to detect variances in the cerebral blood outflow system. The usefulness of positional MR imaging in patients with suspected CCVSI has yet to be investigated.

\section{Acknowledgments}

We thank Emine Taspinar, Gundula Pirch, and. Katharina Huneck for their excellent contribution to this work.

\section{References}

1. Zamboni P, Galeotti R, Menegatti E, et al. Chronic cerebrospinal venous insufficiency in patients with multiple sclerosis. J Neurol Neurosurg Psychiatry 2009;80:392-99

2. Sundstrom $P$, Wahlin A, Ambarki K, et al. Venous and cerebrospinal fluid flow in multiple sclerosis: a case-control study. Ann Neurol 2010;68:255-59

3. Cortes Nino MP, Tampieri D, Melancon D. Endovascular venous procedures for multiple sclerosis? Mult Scler 2010;16:771-72

4. Doepp F, Paul F, Valdueza JM, et al. No cerebrocervical venous congestion in patients with multiple sclerosis. Ann Neurol 2010;68:173-83

5. Stanbrook MB, Hebert PC. Access to treatment for multiple sclerosis must be based on science, not hope. CMAJ 2010;182:1151

6. Epstein HM, Linde HW, Crampton AR, et al. The vertebral venous plexus as a major cerebral venous outflow tract. Anesthesiology 1970;32:332-37

7. Dilenge D, Perey B. An angiographic study of the meningorachidian venous system. Radiology 1973;108:333-37

8. Gisolf J, van Lieshout JJ, van Heusden K, et al. Human cerebral venous outflow pathway depends on posture and central venous pressure. J Physiol 2004;560:317-27

9. Niggemann P, Kuchta J, Grosskurth D, et al. Position dependent changes of the cerebral venous drainage: implications for the imaging of the cervical spine. Cen Eur Neurosurg 2011;72:32-37

10. Alperin N, Hushek SG, Lee SH, et al. MRI study of cerebral blood flow and CSF flow dynamics in an upright posture: the effect of posture on the intracranial compliance and pressure. Acta Neurochir Suppl 2005;95:177-81

11. Zamboni P. Chronic cerebrospinal venous insufficiency. International Angiology 2010;29:91-92

12. Alperin N, Lee SH, Sivaramakrishnan A, et al. Quantifying the effect of posture on intracranial physiology in humans by MRI flow studies. J Magn Reson Imaging 2005;22:591-96

13. Plaisant $\mathrm{O}$, Cosnard G, Gillot $\mathrm{C}$, et al. MRI of the epidural space after gelatin/ gadolinium venous injection. Surg Radiol Anat 1994;16:71-75

14. Chaynes P, Verdie JC, Moscovici J, et al. Microsurgical anatomy of the internal vertebral venous plexuses. Surg Radiol Anat 1998;20:47-51

15. Hoffmann O, Klingebiel R, Braun JS, et al. Diagnostic pitfall: atypical cerebral venous drainage via the vertebral venous system. AJNR Am J Neuroradiol 2002;23:408-11

16. Zamboni P, Galeotti R, Menegatti E, et al. A prospective open-label study of endovascular treatment of chronic cerebrospinal venous insufficiency. $J$ Vasc Surg 2009;50:1348-58 